\title{
Formiato de sódio em dietas para frangos de corte
}

\author{
Sodium formiate in diets for broilers
}

\section{Paula Carvalho Leal ${ }^{\mathrm{I}}$ Sebastião Aparecido Borges ${ }^{\mathrm{II}}$ José Sidney FlemmingIII Everton Luis Krabbe ${ }^{\mathrm{III}}$ Ana Vitória Fischer da Silva ${ }^{\text {III }}$ Alex Maiorka ${ }^{\text {III }}$}

\section{RESUMO}

O estudo foi conduzido para testar a ação do formiato de sódio sobre o desempenho de frangos de corte. No primeiro experimento, foi avaliado o formiato de sódio em níveis crescentes na dieta e, no segundo, a substituição do cloreto de sódio pelo formiato de sódio, com adição de cloreto de amônia para ajuste do balanço eletrolítico. Aos 7, 14 e 21 dias de idade, foram avaliados o consumo de ração, ganho de peso e conversão alimentar e, aos 7 e aos 21 dias, medidos o consumo de água e a umidade das excretas das aves. Os dados obtidos foram submetidos à análise de variância e comparados pelo teste de Tukey. O desempenho de frangos de corte não é afetado quando o formiato de sódio é utilizado em substituição parcial ou total do cloreto de sódio na dieta. Porém, com a adição do formiato de sódio, deve-se observar o nível de sódio e cloro da dieta, de acordo com as exigências das aves.

Palavras chave: aditivos, balanço eletrolítico, cloreto de sódio, consumo de água, umidade de excretas.

\section{ABSTRACT}

This study was developed to evaluate the action of sodium formiate on broiler performance. In the first experiment it was tested the sodium formiate increasing levels in diet, and in the second experiment, it was valuated the replacement of sodium chloride by sodium formiate, with the addition of ammonium chloride for adjustment of electrolyte balance. At 7, 14 and 21 days, feed intake, weight gain and feed conversion were evaluated, and at 7 and 21 days, water intake and excreta moisture were evaluated. The data were submitted to analysis of variance and compared by Tukey test. The performance of broilers is not affected when sodium formate is used in partial or total replacement of sodium chloride in the diet. But with the addition of sodium formate, it should be noted the level of sodium and chlorine in the diet, according to birds' requirement.

Key words: additive, electrolyte balance, excreta moisture, sodium chloride, water intake.

\section{INTRODUÇÃO}

A fonte de sódio $\left(\mathrm{Na}^{+}\right)$mais utilizada na nutrição das aves é o cloreto de sódio $(\mathrm{NaCl})$, porém, é um produto que acelera o processo de corrosão de equipamentos por conta de sua característica higroscópica (ROSA et al., 2010). O formiato de $\mathrm{Na}^{+}$ pode ser uma alternativa ao problema corrosivo, e, além de suplementar a ração com $\mathrm{Na}^{+}$, possui ação antimicrobiana no intestino animal por ser um sal de ácido orgânico derivado do ácido fórmico. Tal ação deve-se ao efeito aniônico do ácido orgânico, sendo esse efeito similar em sais de ácidos orgânicos (no caso, o formiato de $\mathrm{Na}^{+}$) (BELLAVER \& SCHEUERMAN, 2004).

$\mathrm{O} \mathrm{Na}{ }^{+}$, juntamente com o potássio $\left(\mathrm{K}^{+}\right)$e cloro $\left(\mathrm{Cl}^{-}\right)$são eletrólitos fundamentais na manutenção da pressão osmótica dos líquidos corporais e equilíbrio ácido-básico do organismo animal. São exigidas quantidades mínimas nas dietas das aves para que suas necessidades nutricionais sejam alcançadas e suas corretas proporções mantidas (SILVA et al., 2009a).

'Programa de Pós-graduação em Ciências Veterinárias, Universidade Federal do Paraná (UFPR), 80060-000, Curitiba, PR, Brasil.

E-mail: paulacarvalholeal@gmail.com. Autor para correspondência.

"Iniversidade Tuiuti do Paraná (UTP), Curitiba, PR, Brasil.

IIIUFPR, Curitiba, PR, Brasil. 
A suplementação de $\mathrm{Na}^{+}, \mathrm{Cl}^{-}$e $\mathrm{K}^{+}$torna-se fundamental para a preservação das funções fisiológicas e alcance do desempenho zootécnico desejado.

A equação: $\mathrm{Na}^{+}+\mathrm{K}^{+}-\mathrm{Cl}^{-}$, proposta por MONGIN \& SAUVEUR (1977), expressa o balanço eletrolítico (BE), que expõe a quantidade e relação entre esses minerais, sendo apresentado nas dietas em miliequivalentes por quilo de ração $\left(\mathrm{mEq} \mathrm{kg}^{-1}\right)$. O cloreto de amônia $\left(\mathrm{NH}_{4} \mathrm{Cl}\right)$ é um exemplo de ingrediente que pode ser utilizado para que o BE se mantenha adequado.

O presente trabalho foi realizado para testar a inclusão do formiato de sódio e a substituição do $\mathrm{NaCl}$ na dieta, e seus efeitos no desempenho zootécnico, consumo de água e umidade das excretas de frangos de corte criados de 1 a 21 dias de idade.

\section{MATERIAL E MÉTODOS}

Foram realizados dois experimentos, cada um utilizando 300 pintos de corte da linhagem comercial Ross, machos e com peso médio inicial de 45 gramas. As aves foram criadas de 1 a 21 dias de idade em baterias metabólicas divididas em gaiolas de $0,98 \times 0,90 \times 0,50 \mathrm{~cm}$ (c x $1 \times$ h), recebendo rações formuladas à base de milho e farelo de soja, utilizando níveis nutricionais recomendados por ROSTAGNO et al. (2005) (Tabela 1). $\mathrm{O}$ delineamento empregado foi inteiramente casualizado, em que cada experimento foi composto por cinco tratamentos de seis repetições com 10 aves cada, sendo os tratamentos divididos de acordo com o nível de adição do formiato de $\mathrm{Na}^{+}$e composição eletrolítica das dietas empregadas, conforme apresentado na tabela 2 .

No experimento 1 , foi testado o efeito da inclusão de níveis crescentes de formiato de $\mathrm{Na}^{+}$, enquanto no experimento 2 , foi feita a substituição do $\mathrm{NaCl}$ pelo formiato de $\mathrm{Na}^{+}$, mas com adição de $\mathrm{NH}_{4} \mathrm{Cl}$ com o intuito de manter constante o nível de $\mathrm{Cl}^{-}$entre os tratamentos. O desempenho zootécnico foi determinado em ambos, por meio da pesagem das aves e das rações ao alojamento, aos 7, 14 e 21 dias de idade, para avaliação do consumo de ração (CR), ganho de peso (GP) e conversão alimentar (CA).

Aos 7 e aos 21 dias, foram avaliados o consumo de água $\left(\mathrm{ConH}_{2} \mathrm{O}\right)$ e a umidade das excretas (U $105^{\circ}$ ) dos animais. Para determinação de umidade de excretas, as gaiolas dispunham de bandejas sob elas, previamente revestidas com plástico para assegurar que a coleta total de excretas fosse feita conforme metodologia descrita por SAKOMURA \& ROSTAGNO (2007), e as análises laboratoriais segundo método de SILVA \& QUEIROZ (2002).

Os dados obtidos foram submetidos à análise de variância (ANOVA) e, ocorrendo diferenças
Tabela 1 - Dietas experimentais fornecidas a frangos de corte de 1 a 21 dias de idade.

\begin{tabular}{|c|c|}
\hline Ingredientes & Inclusão (\%) \\
\hline Milho & 54,78 \\
\hline Farelo de soja & 35,34 \\
\hline Óleo de soja & 4,71 \\
\hline Fosfato bicálcico & 1,84 \\
\hline Calcário & 0,95 \\
\hline Premix vitamínico ${ }^{1}$ & 0,30 \\
\hline Premix mineral $^{2}$ & 0,20 \\
\hline DL- Metionina & 0,25 \\
\hline L- Lisina $(\mathrm{HCl})$ & 0,18 \\
\hline Espaço vazio $^{3}$ & 1,45 \\
\hline Proteína bruta $(\%)$ & 21,00 \\
\hline Energia metabolizável $\left(\mathrm{kcal} \mathrm{kg}^{-1}\right)$ & 3.050 \\
\hline Cálcio $(\%)$ & 0,95 \\
\hline Fósforo disponível (\%) & 0,45 \\
\hline Lisina digestível (\%) & 0,15 \\
\hline Metionina digestível (\%) & 0,45 \\
\hline Met. + Cist. digestível (\%) & 0,81 \\
\hline Treonina digestível (\%) & 0,70 \\
\hline Triptofano digestível (\%) & 0,19 \\
\hline
\end{tabular}

${ }^{1}$ Fornecido por kg de ração: vitamina A (11.000UI), vitamina D3 (2.250UI), vitamina E (9UI), vitamina $\mathrm{K} 3(1,8 \mathrm{mg})$, vitamina B12 $(0,012 \mathrm{mg})$, tiamina $(1,1 \mathrm{mg})$, riboflavina $(9 \mathrm{mg})$, piridoxina $(1,8 \mathrm{mg})$, biotina $(0,1 \mathrm{mg})$, colina $(680 \mathrm{mg})$, ácido pantotênico (9mg), niacina (35mg), ácido fólico (1mg).

${ }^{2}$ Fornecido por kg de ração: selênio $(0,18 \mathrm{mg})$, iodo $(1,2 \mathrm{mg})$, ferro (70mg), cobre (10mg), zinco (60mg), manganês (70mg).

${ }^{3}$ Varia de acordo com os tratamentos.

significativas, comparados estatisticamente pelo teste de Tukey $(\mathrm{P}<0,05)$.

\section{RESULTADOS E DISCUSSÃO}

Aos sete dias de idade, as aves do tratamento controle, sem adição de formiato de $\mathrm{Na}^{+}$, apresentaram consumo de ração semelhante ao tratamento $2(\mathrm{P}>0,05)$, em que a inclusão de formiato foi a menor entre os tratamentos que o receberam $(0,580 \%)$, e diferiram dos demais $(\mathrm{P}<0,05)$, nos quais houve queda de consumo de ração (Tabela 3 ).

O ganho de peso das aves também foi reduzido significativamente nos tratamentos em que houve adição de formiato de $\mathrm{Na}^{+}$. $\mathrm{O}$ tratamento 2 $\left(0,580 \%\right.$ de formiato de $\left.\mathrm{Na}^{+}\right)$foi o que apresentou menor ganho de peso quando confrontado com o tratamento 1 (controle, sem formiato). A conversão alimentar das aves foi da mesma forma afetada pela adição de formiato de $\mathrm{Na}^{+}$às dietas, sendo o pior índice, também observado no tratamento 2. Essa constatação pode estar associada ao menor nível de $\mathrm{Na}^{+}$e $\mathrm{Cl}^{-}(0,13 \%$ e $0,07 \%$, 
Tabela 2 - Variação da composição eletrolítica das dietas experimentais.

\begin{tabular}{|c|c|c|c|c|c|}
\hline Ingrediente & $\mathrm{T} 1$ & $\mathrm{~T} 2$ & $\mathrm{~T} 3$ & $\mathrm{~T} 4$ & T5 \\
\hline Formiato de sódio (\%) & 0,000 & 0,580 & 0,825 & 1,090 & 1,350 \\
\hline Cloreto de sódio $(\mathrm{NaCl}, \%)$ & 0,410 & 0,000 & 0,000 & 0,000 & 0,000 \\
\hline Cloreto de amônia $\left(\mathrm{NH}_{4} \mathrm{Cl}, \%\right)$ & - & - & - & - & - \\
\hline Inerte $(\%)$ & 1,04 & 0,87 & 0,625 & 0,360 & 0,100 \\
\hline Sódio $\left(\mathrm{Na}^{+}, \%\right)$ & 0,21 & $\begin{array}{l}\text { sçao ca } \\
0,13\end{array}$ & 0,17 & 0,21 & 0,25 \\
\hline Potássio $\left(\mathrm{K}^{+}, \%\right)$ & 0,80 & 0,80 & 0,80 & 0,80 & 0,80 \\
\hline Cloro $\left(\mathrm{Cl}^{-}, \%\right)$ & 0,28 & 0,07 & 0,09 & 0,11 & 0,12 \\
\hline $\mathrm{BE}^{*}\left(\mathrm{mEq} \mathrm{kg}{ }^{-1}\right)$ & 213 & 238 & 251 & 264 & 277 \\
\hline Ingrediente & $\mathrm{T} 1$ & $\mathrm{~T} 2$ & T3 & $\mathrm{T} 4$ & T5 \\
\hline Formiato de sódio (\%) & 0,000 & 0,280 & 0,550 & 0,820 & 1,090 \\
\hline Cloreto de sódio $(\mathrm{NaCl}, \%)$ & 0,410 & 0,310 & 0,200 & 0,100 & 0,000 \\
\hline Cloreto de amônia $\left(\mathrm{NH}_{4} \mathrm{Cl}, \%\right)$ & 0,000 & 0,090 & 0,180 & 0,270 & 0,360 \\
\hline Inerte $(\%)$ & 1,040 & 0,770 & 0,520 & 0,260 & - \\
\hline Sódio $\left(\mathrm{Na}^{+}, \%\right)$ & 0,21 & $\begin{array}{l}\text { ição ca } \\
0,21\end{array}$ & 0,21 & 0,21 & 0,21 \\
\hline Potássio $\left(\mathrm{K}^{+}, \%\right)$ & 0,80 & 0,80 & 0,80 & 0,80 & 0,80 \\
\hline Cloro $\left(\mathrm{Cl}^{-}, \%\right)$ & 0,28 & 0,30 & 0,31 & 0,33 & 0,34 \\
\hline $\mathrm{BE}^{*}\left(\mathrm{mEq} \mathrm{kg}{ }^{-1}\right)$ & 213 & 210 & 205 & 201 & 197 \\
\hline
\end{tabular}

$\mathrm{BE}^{*}=$ Balanço Eletrolítico

respectivamente), causando maior dificuldade da manutenção do equilíbrio ácido-básico da dieta (238mEq) em relação aos demais. O efeito da suplementação de níveis crescentes de $\mathrm{Na}^{+}$na forma de $\mathrm{NaCl}$, para frangos de corte até sete dias de idade, sugere que a exigência deste eletrólito na primeira semana de vida das aves é de aproximadamente 0,39\% (BRITON, 1992).

Aos 14 dias, as aves dos tratamentos que receberam o formiato de $\mathrm{Na}^{+}$(T2, T3, T4, T5) apresentaram menor consumo de ração quando confrontados com o tratamento controle (T1), sem formiato. A causa para tal resultado, de acordo com MONGIN (1981), seria um possível desequilíbrio ácidobásico influenciado pelos íons de $\mathrm{Na}^{+}, \mathrm{K}^{+} \mathrm{e} \mathrm{Cl}$. Também foram observados efeitos negativos sobre o ganho de peso e a conversão alimentar das aves dos tratamentos em que o formiato foi adicionado. O NRC (1994) recomenda valores iguais a $0,30 \%, 0,20 \%$ e $0,20 \%$ para $\mathrm{K}^{+}, \mathrm{Na}^{+}$e $\mathrm{Cl}^{-}$, respectivamente, no período de 0 a 3 semanas de idade.

Com 21 dias, o menor consumo de ração foi observado nas aves do tratamento $2(0,580 \%$ de formiato) quando comparado ao tratamento 1 (sem inclusão de formiato) e ao tratamento $5(1,350 \%$ de formiato). $\mathrm{O}$ ganho de peso das aves novamente foi afetado negativamente quando receberam formiato de $\mathrm{Na}^{+}$nos tratamentos $\mathrm{T} 2, \mathrm{~T} 3, \mathrm{~T} 4$ e T5, em que os índices de balanço eletrolítico foram de 238, 251, 264 e $277 \mathrm{mEq}$ $\mathrm{kg}^{-1}$, respectivamente.

A conversão alimentar das aves do tratamento 1 (sem formiato com $21 \% \mathrm{de} \mathrm{Na}^{+}$) foi melhor que a apresentada pelas aves do tratamento 2 e tratamento $5\left(0,13\right.$ e $0,25 \%$ de $\mathrm{Na}^{+}$, respectivamente), não diferindo dos demais. Segundo BARROS et al. (2001), a exigência nutricional mínima de $\mathrm{Na}^{+}$para um bom ganho de peso e conversão alimentar de frangos de corte de 1 a 21 dias é de $0,256 \%$.

$\mathrm{O}$ fato de os animais terem uma necessidade mínima para cada eletrólito e o emprego de dietas em níveis marginais de $\mathrm{K}^{+}, \mathrm{Na}^{+}$e $\mathrm{Cl}^{-}$pode gerar respostas negativas para o desempenho zootécnico. Assim, os níveis de $\mathrm{Cl}^{-}$nos tratamentos com formiato de $\mathrm{Na}^{+}$podem estar abaixo do necessário para o máximo desempenho das aves, explicando o efeito negativo da suplementação desse aditivo nas rações. A importância da relação entre $\mathrm{K}^{+}, \mathrm{Na}^{+}$e $\mathrm{Cl}^{-}$é reforçada por BORGES et al. (2003), que ressalta a necessidade de que sejam atendidas as exigências nutricionais dos animais para esses eletrólitos. Esses resultados podem ser melhor observados no experimento 2 deste estudo, em que houve correção dos níveis de $\mathrm{Cl}^{-}$das dietas utilizando $\mathrm{NH}_{4} \mathrm{Cl}$.

As avaliações de consumo de água e umidade das excretas das aves, também do experimento 1, são apresentados na tabela 3. Aos sete dias, a adição 
Formiato de sódio em dietas para frangos de corte.

Tabela 3 - Efeito da inclusão de níveis crescentes de formiato de sódio sobre o consumo de ração (CR), ganho de peso (GP), conversão alimentar (CA) aos 7, 14 e 21 dias, e consumo de água $\left(\mathrm{ConH}_{2} \mathrm{O}\right)$ e umidade das excretas a $105^{\circ} \mathrm{C}\left(\mathrm{U} 105^{\circ}\right)$ aos 7 e 21 dias de idade de frangos de corte.

\begin{tabular}{|c|c|c|c|c|c|c|c|}
\hline & $\mathrm{T} 1$ & $\mathrm{~T} 2$ & $\mathrm{~T} 3$ & $\mathrm{~T} 4$ & T5 & & \\
\hline Formiato de sódio (\%) & 0,000 & 0,580 & 0,825 & 1,090 & 1,350 & & \\
\hline \multirow[t]{2}{*}{ Cloreto de sódio (\%) } & 0,410 & 0,000 & 0,000 & 0,000 & 0,000 & & \\
\hline & \multicolumn{5}{|c|}{------Período de 1 a 7 dias---------- } & $\mathrm{CV}(\%)$ & $\mathrm{P}=$ \\
\hline $\mathrm{CR}(\mathrm{g})$ & $154^{\mathrm{a}}$ & $147^{\mathrm{ab}}$ & $146^{\mathrm{b}}$ & $144^{\mathrm{b}}$ & $144^{\mathrm{b}}$ & 4,09 & 0,017 \\
\hline GP $(g)$ & $134^{\mathrm{a}}$ & $110^{\mathrm{c}}$ & $118^{\mathrm{bc}}$ & $118^{\mathrm{bc}}$ & $121^{\mathrm{b}}$ & 7,88 & 0,001 \\
\hline $\mathrm{CA}\left(\mathrm{g} \mathrm{g}^{-1}\right)$ & $1,18^{\mathrm{a}}$ & $1,34^{\mathrm{c}}$ & $1,24^{\mathrm{b}}$ & $1,22^{\mathrm{ab}}$ & $1,19^{\mathrm{ab}}$ & 6,32 & 0,001 \\
\hline $\mathrm{ConH}_{2} \mathrm{O}(\mathrm{g})$ & $42^{\mathrm{a}}$ & $31^{\mathrm{b}}$ & $32^{\mathrm{b}}$ & $31^{\mathrm{b}}$ & $36^{\mathrm{b}}$ & 15,77 & 0,001 \\
\hline \multirow{2}{*}{$\mathrm{U} 105^{\circ}(\%)$} & 73,8 & 73,3 & 71,1 & 71,3 & 73,9 & 16,60 & 0,442 \\
\hline & \multicolumn{4}{|c|}{------Período de 1 a 14 dias--------- } & ------ & $\mathrm{CV}(\%)$ & $\mathrm{P}=$ \\
\hline $\mathrm{CR}(\mathrm{g})$ & $539^{a}$ & $513^{\mathrm{b}}$ & $505^{\mathrm{c}}$ & $503^{\mathrm{c}}$ & $487^{\mathrm{c}}$ & 4,39 & 0,001 \\
\hline $\mathrm{GP}(\mathrm{g})$ & $453^{\mathrm{a}}$ & $411^{\mathrm{b}}$ & $399^{\mathrm{b}}$ & $398^{\mathrm{b}}$ & $361^{\mathrm{c}}$ & 8,21 & 0,001 \\
\hline \multirow[t]{2}{*}{$\mathrm{CA}\left(\mathrm{g} \mathrm{g}^{-1}\right)$} & $1,19^{\mathrm{a}}$ & $1,35^{\mathrm{b}}$ & $1,27^{\mathrm{b}}$ & $1,24^{\mathrm{b}}$ & $1,25^{\mathrm{b}}$ & 4,76 & 0,001 \\
\hline & \multicolumn{4}{|c|}{----Período de 1 a 21 dias--- } & ----- & $\mathrm{CV}(\%)$ & $\mathrm{P}=$ \\
\hline $\mathrm{CR}(\mathrm{g})$ & $1133^{\mathrm{a}}$ & $1017^{\mathrm{b}}$ & $1078^{\mathrm{ab}}$ & $1074^{\mathrm{ab}}$ & $1105^{\mathrm{a}}$ & 5,08 & 0,001 \\
\hline $\mathrm{GP}(\mathrm{g})$ & $930^{\mathrm{a}}$ & $776^{\mathrm{c}}$ & $860^{\mathrm{b}}$ & $857^{\mathrm{b}}$ & $860^{\mathrm{b}}$ & 7,11 & 0,001 \\
\hline $\mathrm{CA}\left(\mathrm{g} \mathrm{g}^{-1}\right)$ & $1,22^{\mathrm{a}}$ & $1,31^{\mathrm{c}}$ & $1,25^{\mathrm{ab}}$ & $1,25^{\mathrm{ab}}$ & $1,28^{\mathrm{bc}}$ & 3,22 & 0,001 \\
\hline $\mathrm{ConH}_{2} \mathrm{O}(\mathrm{g})$ & $176^{\mathrm{ab}}$ & $161^{\mathrm{b}}$ & $189^{\mathrm{a}}$ & $178^{\mathrm{a}}$ & $182^{\mathrm{a}}$ & 8,47 & 0,005 \\
\hline $\mathrm{U} 105^{\circ}(\%)$ & $75,2^{\mathrm{ab}}$ & $74,3^{\mathrm{b}}$ & $76,7^{\mathrm{ab}}$ & $76,8^{\mathrm{ab}}$ & $78,4^{\mathrm{a}}$ & 3,17 & 0,023 \\
\hline
\end{tabular}

Médias seguidas por letras minúsculas distintas, na mesma linha, diferem significativamente entre si pelo teste de Tukey (P<0,05).

CV: Coeficiente de variação.

P: Probabilidade.

de formiato de $\mathrm{Na}^{+}$às dietas em níveis crescentes levou ao menor consumo de água das aves. Porém, não foram observados efeitos dos níveis de inclusão sobre a umidade das excretas.

Aos 21 dias, as aves do tratamento 2 $\left(0,580 \%\right.$ de formiato de $\left.\mathrm{Na}^{+}\right)$apresentaram consumo de água inferior às aves dos demais tratamentos que receberam formiato de $\mathrm{Na}^{+}$em maior proporção (T3, T4 e T5). O motivo para tal resultado pode ser a menor quantidade de $\mathrm{Na}^{+}$no tratamento 2, o que se encontra em consonância com os resultados observados por BORGES et al. (1999), que encontraram aumento linear no consumo de água de acordo com o aumento da relação eletrolítica quando o eletrólito manipulado foi o $\mathrm{Na}^{+}$, uma vez que a ave tenta satisfazer a sensação de sede criada pela alta ingestão de $\mathrm{Na}^{+}$.

Para umidade das excretas das aves, o tratamento $2(0,580 \%$ de formiato $)$ obteve o menor valor $(\mathrm{P}<0,05)$ quando confrontado com o tratamento 5 (1,350\% de formiato), sendo os demais estatisticamente semelhantes. $\mathrm{O}$ teor de umidade das excretas se eleva com o aumento do balanço eletrolítico das dietas, já que alterações na quantidade de $\mathrm{Na}^{+}$e $\mathrm{K}^{+}$aumentam o consumo de água (OLIVEIRA et al., 2010).

$\mathrm{O}$ experimento 2 tem seus dados apresentados na tabela 4, verificando-se que nenhuma das variáveis avaliadas apresentaram diferenças significativas sobre o desempenho das aves $(\mathrm{P}>0,05)$.
Resultados semelhantes foram encontrados por SILVA et al. (2009a) e SILVA et al. (2009b), que, avaliando fontes de $\mathrm{Na}^{+}$, constataram que o desempenho das aves que receberam dietas com adição de formiato de $\mathrm{Na}^{+}$não foi comprometido. Isto evidencia que, desde que corrigidos os níveis dos eletrólitos totais das dietas, o formiato de $\mathrm{Na}^{+}$poderia substituir o $\mathrm{NaCl}$ como fonte de $\mathrm{Na}^{+}$para frangos de corte.

Assim como na avaliação de desempenho zootécnico, sobre o consumo de água e a umidade das excretas das aves no experimento 2 , também não foram observadas diferenças entre os tratamentos (Tabela 4). Esse resultado está de acordo com os obtidos por SILVA et al. (2009a), que não verificaram aumento de umidade das excretas, consequentemente, da umidade da cama das aves que receberam $\mathrm{NaCl}$ e formiato de $\mathrm{Na}^{+}$. Além disso, a inclusão de $\mathrm{NH}_{4} \mathrm{Cl}$ ao tratamento contendo formiato de $\mathrm{Na}^{+}$não trouxe alterações significativas à umidade das excretas.

\section{CONCLUSÃO}

O desempenho de frangos de corte não é afetado quando o formiato de sódio é utilizado em substituição parcial ou total do cloreto de sódio na dieta. Porém, com a adição do formiato de sódio, devese observar o nível de sódio e cloro da dieta, de acordo com as exigências das aves. 
Tabela 4 - Efeitos da substituição do cloreto de sódio pelo formiato de sódio sobre o consumo de ração (CR), ganho de peso (GP), conversão alimentar (CA) aos 7, 14 e 21 dias, e consumo de água $\left(\mathrm{ConH}_{2} \mathrm{O}\right)$ e umidade das excretas a $105^{\circ} \mathrm{C}\left(\mathrm{U} 105^{\circ}\right)$ aos 7 e 21 dias de idade de frangos de corte.

\begin{tabular}{|c|c|c|c|c|c|c|c|}
\hline & $\mathrm{T} 1$ & $\mathrm{~T} 2$ & $\mathrm{~T} 3$ & $\mathrm{~T} 4$ & T5 & & \\
\hline Formiato de sódio (\%) & 0,000 & 0,280 & 0,550 & 0,820 & 1,090 & & \\
\hline Cloreto de sódio (\%) & 0,410 & 0,310 & 0,200 & 0,100 & 0,000 & & \\
\hline \multirow{2}{*}{ Cloreto de amônia (\%) } & 0,000 & 0,090 & 0,180 & 0,270 & 0,360 & & \\
\hline & \multicolumn{3}{|c|}{---Período de 1 a 7 dias--- } & & ------ & $\mathrm{CV}(\%)$ & $\mathrm{P}=$ \\
\hline $\mathrm{CR}(\mathrm{g})$ & 173 & 173 & 177 & 177 & 1973 & 3,57 & 0,581 \\
\hline $\mathrm{GP}(\mathrm{g})$ & 147 & 148 & 152 & 149 & 145 & 6,46 & 0,781 \\
\hline $\mathrm{CA}\left(\mathrm{g} \mathrm{g}^{-1}\right)$ & 1,18 & 1,17 & 1,17 & 1,19 & 1,20 & 5,05 & 0,858 \\
\hline $\mathrm{ConH}_{2} \mathrm{O}(\mathrm{g})$ & 240 & 247 & 252 & 242 & 244 & 6,04 & 0,709 \\
\hline \multirow{2}{*}{$\mathrm{U} 105^{\circ}(\%)$} & 71,9 & 71,9 & 72,0 & 72,4 & 71,3 & 3,31 & 0,952 \\
\hline & \multicolumn{3}{|c|}{--Período de 1 a 14 dias---- } & & ------- & $\mathrm{CV}(\%)$ & $\mathrm{P}=$ \\
\hline CR $(g)$ & 572 & 574 & 581 & 583 & 582 & 2,43 & 0,554 \\
\hline GP $(g)$ & 467 & 471 & 473 & 472 & 461 & 3,14 & 0,625 \\
\hline \multirow{2}{*}{$\mathrm{CA}\left(\mathrm{g} \mathrm{g}^{-1}\right)$} & 1,22 & 1,22 & 1,23 & 1,24 & 1,26 & 2,38 & 0,057 \\
\hline & \multicolumn{3}{|c|}{--Período de 1 a 21 dias--- } & & ----- & $\mathrm{CV}(\%)$ & $\mathrm{P}=$ \\
\hline CR $(g)$ & 1335 & 1339 & 1320 & 1323 & 1332 & 2,16 & 0,781 \\
\hline $\mathrm{GP}(\mathrm{g})$ & 943 & 954 & 934 & 915 & 914 & 3,97 & 0,264 \\
\hline $\mathrm{CA}\left(\mathrm{g} \mathrm{g}^{-1}\right)$ & 1,42 & 1,4 & 1,41 & 1,45 & 1,46 & 3,22 & 0,201 \\
\hline $\mathrm{ConH}_{2} \mathrm{O}(\mathrm{g})$ & 212 & 224 & 204 & 222 & 213 & 8,87 & 0,386 \\
\hline $\mathrm{U} 105^{\circ}(\%)$ & 77,5 & 77,1 & 77,2 & 77,4 & 77,2 & 1,47 & 0,970 \\
\hline
\end{tabular}

Médias seguidas por letras minúsculas distintas, na mesma linha, diferem significativamente entre si pelo teste de Tukey $(\mathrm{P}<0,05)$.

CV: Coeficiente de variação.

P: Probabilidade.

\section{COMITÊ DE ÉTICA E BIOSSEGURANÇA}

Número do protocolo 028/2006

\section{REFERÊNCIAS}

BARROS, J. M. S. et al. Exigência nutricional de sódio para frangos de corte de 1 a 21 dias de idade. Revista Brasileira de Zootecnia, v.30, n.3, p.1044-1051, 2001. Disponível em: <http://www.scielo.br/pdf/rbz/v30n3s1/5524.pdf >. Acesso em: 23 mar. 2011.

BELLAVER, C.; SCHEUERMANN, G. Aplicações dos ácidos orgânicos na produção de aves de corte. In: CONFERÊNCIA AVESUI, 2004, Florianópolis, SC. Anais... Florianópolis: [s.n.], 2004. p.1-16.

BORGES, S. A. et al. Balanço eletrolítico em dietas pré-iniciais de frangos de corte durante o verão. Revista Brasileira de Ciência Avícola, v.1, n.3, p.175-179, 1999. Disponível em: <http://www.qgncarbonor.com.br/includes/arquivos/artigos/ nutricaoanimal/monogastricos/bepreinicial.pdf $>$. Acesso em: 23 mar. 2011.

BORGES, S. A. et al. Fisiologia do estresse calórico e a utilização de eletrólitos em frangos de corte. Ciência Rural, v.33, n.5, p.975-981, 2003. Disponível em: <http://www.scielo.br/pdf/cr/ v33n5/17148.pdf>. Acesso em: 23 mar. 2011.

BRITON, W. M. Effect of dietary salt intake on water and feed consumption. In: NUTRITION PROCEEDINGS CONFERENCE FOR FEED INDUSTRY, 1992, Athens, GA. Anais... Local: University of Geórgia, 1992. p.48-53.
MONGIN, P.; SAUVEUR, B. Interrelationships between mineral nutrition, acid-base balance, growth and cartilage abnormalities. In: GROWTH AND POULTRY MEAT PRODUCTION, 1977, Edinburgh. Proceedings Poultry Science. Edinburgh: British Poultry Science, 1977. N.12, p.235-247.

MONGIN, P. Recent advances in dietary anion-cation balance in poultry. Recent Advances in Animal Nutrition, PublButterworths, v.40, p.109-119, 1981. Disponível em: < h t t p : / / j o u r n a $1 \mathrm{~s}$. c a m b r i g e.org / download.php?file=\%2FPNS\%2FPNS40_03\%2FS002966518 1000499a.pdf \&code $=$ ef9a39e 73ed998089f1f376d4cb1 ef35> . Acesso em: 23 mar. 2011.

NRC (National Research Council). Nutrient Requirements of Poultry. 9.rev.ed. Washington, DC: Nacional Academy, 1994. 155p.

OLIVEIRA, M. C. et al. Efeito do balanço eletrolítico da ração sobre parâmetros ósseos e da cama de frango. Revista Biotemas, v.23, n.1, p.203-209, 2010. Disponível em: <http:/ /www.biotemas.ufsc.br/volumes/pdf/volume231/203a209.pdf>. Acesso em: 23 mar. 2011.

ROSA, E. R. et al. Desempenho e rendimento de carcaça de frangos alimentados com diferentes fontes de sódio. Ciência Animal Brasileira, v.11, n.1, p.73-79, 2010. Disponível em: <http://www.revistas.ufg.br/index.php/vet/article/download/ 1421/6510>. Acesso em: 23 mar. 2011. doi: 10.5216/ cab.v11i1.1421.

ROSTAGnO, H. S. et al. Tabelas brasileiras para aves e suínos: composição de alimentos e exigências nutricionais. 3ed. Viçosa, MG: UFV, 2005. 141p. 
SAKOMURA, N. K.; ROSTAGNO, H.S. Métodos de pesquisa em nutrição de monogástricos. Jaboticabal: FUNEP, 2007. $283 \mathrm{p}$.

SILVA, D. J.; QUEIROZ, A.C. Análise de alimentos: métodos químicos e biológicos. 3.ed. Viçosa: UFV, 2002. 235p.

SILVA, R. A. G. et al. Uso de diferentes fontes de sódio na dieta de frangos de corte de 22 a 42 dias de idade sobre o desempenho e umidade da cama. Ciência Animal Brasileira, v.10, n.4, p.1029-1036, 2009a. Disponível em: <http://www.revistas.ufg.br/ index.php/vet/article/viewFile/2680/5916>. Acesso em: 23 mar. 2011.

SILVA, R. A. G. et al. Diferentes níveis de formiato de sódio em substituição ao cloreto de sódio na dieta de frangos de corte. Journal Acta Scientiarum Animal Sciences, v.31, n.3, p.251255, 2009b. Disponível em: <http://periodicos.uem.br/ojs/ index.php/ActaSciAnimSci/article/viewFile/6020/6020>. Acesso em: 23 mar. 2011. doi: 10.4025/actascianimsci.v31i3.6020. 